\title{
Comparison of the energy spectra between pileup shock and converging shock
}

\author{
Xin Wang, ${ }^{a, b, c, f,{ }^{*}}$ Xueshang Feng, ${ }^{b}$ Yihua Yan, ${ }^{c}$ Mingde Ding, ${ }^{d}$ Hong Lu ${ }^{e}$ and Hao \\ Shan $^{a, f}$ \\ ${ }^{a}$ Xinjiang Astronomical Observatory, Chinese Academy of Sciences, \\ 830011, Urumqi, China \\ ${ }^{b}$ State Key Laboratory of Space Weather, Chinese Academy of Sciences, \\ 100190, Beijing, China \\ ${ }^{c}$ CAS Key Laboratory of Solar Activity, NAOC, \\ 100012, Beijing, China \\ ${ }^{d}$ Key Laboratory of Modern Astronomy and Astrophysics (Nanjing University), Ministry of Education, \\ 210093, Nanjing, China \\ ${ }^{e}$ Key Laboratory of Particle Astrophysics, Institute of High Energy Physics, Chinese Academy of Sciences, \\ 100049, Beijing, China \\ ${ }^{f}$ Key Laboratory of Radio Astronomy, Chinese Academy of Sciences, \\ 210008, Nanjing, China \\ E-mail: wangxin@xao.ac.cn
}

We present a few possibilities for forming an extended energy spectrum and producing a varied slope in different double-shock models. In our previous work, the converging double-shock model would provide more kinetic energy injecting into the particles acceleration. The high efficient injection rate excited by amplified magnetic turbulence in the converged region made the extended energy spectrum be possible. In our present work, the pileup-shock model provides an opportunity of the re-accelerated processes of the particles on the merged precursor region. With the expending of the precursor region, more and more particles can be participated into the pileup-shock system, the "ankle" slope of the energy spectrum would be produced due to the enhancement magnetic turbulence between the merged pileup-shock. We have proved that the converging double-shock model taken a negative effect on the accelerated particles and produce an energy "break" slope. At present, we also investigate that a positive effect on particle acceleration in pileup twin-shock scenario can produce an "ankle" slope on the energy spectrum.

$37^{\text {th }}$ International Cosmic Ray Conference (ICRC 2021)

July 12th - 23rd, 2021

Online - Berlin, Germany

\footnotetext{
*Presenter
} 


\section{Introduction}

Observations show that there is a proton spectral "break" with $\mathrm{E}_{\text {break }}$ at $1-10 \mathrm{MeV}$ in some large CME-driven shocks[18]. Theoretical model usually attribute this phenomenon to a diffusive shock acceleration. However, the underlying physics of the shock acceleration still remains uncertain. Although previous numerical models can hardly predict this "break" due to either high computational expense or shortcomings of current models, our previous paper [12] focused on simulating this energy spectrum in converged double-shock by Monte Carlo numerical method. Considering the Dec 132006 CME-driven shock interaction with an Earth bow shock, we have unexpectedly found a proton spectral "break" appeared distinctly at the energy $\sim 5 \mathrm{MeV}$.

Although there is still no reliable prediction of this "break" by numerical methods, Monte Carlo (MC) method can easily treat thermal ion injection $[8,9]$. In MC method, the scattering mean free path is assumed to be the function of the particle rigidity, so this treatment is able to follow the evolution of individual ions long enough to model acceleration to high energies. Ellison et al. [15] presented an ion spectra with a maximum particle energy no more than $1 \mathrm{MeV}$ by applying an fixed free escape boundary(FEB) ahead of the bow shock. Knerr et al. [16] and Wang et al. [10] forward the maximum particle energy achieving to $\sim 4 \mathrm{MeV}$ by applying a moving FEB with the shock. Wang et al. [11] investigated that the maximum particle energy limited in FEB can achieve a saturation at $\sim 5.5 \mathrm{MeV}$ using different scattering mean free path functions.

Ground-level enhancement (GLE) events are one type of large gradual SEP event. Upon examining all 16 GLEs in solar cycle 23, Li et al. [4] proposed a "twin-CME" scenario for GLE events and large SEP events. In this scenario, two CMEs go off closely in time from the same active region (AR). The first CME drives a shock which generates a very turbulent downstream. The first shock can also pre-accelerate particles, although not necessarily to very high energies. As the second CME plunges into the strong turbulent region downstream of the first shock with the pre-accelerated population, it will accelerate them to very high energies.

Sympathetic eruptions are multiple eruptions within a relatively short period of time, which may occur across different source regions, even more cover a full hemisphere, or in a single complex source region $[1,3,5,6,14]$. If two sympathetic eruptions from the same AR occur close in time and both drive shocks, then they are in agreement with the "twin CME" scenario and therefore can be candidate sites for efficient particle accelerations.

The "twin CME" scenario focusing on the presence of two CME-driven shocks, resembles that of sympathetic eruptions. Here, we called it as the pileup shock model. Because the "twin CME" scenario suggests that efficient acceleration occurs at the shock driven by the second CME, it implies that a single CMEs may not lead to large SEP events. Wang et al. [11] have examined an isolated CME-driven shock could accelerate the proton to the largest attainable energies of SEP events up to $\sim 5 \mathrm{MeV}$. Ding et al. [2] tested the "twin CME" scenario against all large SEP events and western CMEs with speed $>900 \mathrm{~km} / \mathrm{s}$ in solar cycle 23 and found that most of the large SEP events agree with the "twin CME scenario and many single fast CMEs do not lead to large SEP events. Wang et al. [12] investigated the interactions between the CME-driven shock and Earth's bow shock, which could produce the highest attainable proton energy reaching to $\sim 20 \mathrm{MeV}$, but with a soft energy spectral slope beyond the "break" point at $\sim 5.5 \mathrm{MeV}$. At present, we propose a simulation of the pileup shock interactions for further investigating a high energy "tail" and the 
possible energy spectral variation.

Wang et al. [13] suggest that there were two eruptions and two CMEs in the 2012 May 17 event. Both CMEs were fast and were clearly seen from STEREO-B coronagraph observation. Multiple episodes of type II radio bursts were also observed confirming the existence of CME-driven shocks. The observed ground-level enhancement of energetic particles in the 2012 May 17 GLE event and the fact that there were two fast CMEs in the event supports the recently proposed "twin CME" scenario for GLE events.

\section{Model 1}

Halo CMEs were observed by the SOHO/LASCO coronagraphs in association with the events of 13 December 2006, with speeds of $1774 \mathrm{kms}^{-1}$. The flux spectra of protons in the SEP event by ACE, STEREO, and SAMPEX instruments show a "break" at $\sim 1-10 \mathrm{MeV}$. Spectra from GOES-11 also agree over the region from $5 \mathrm{MeV}$ to $100 \mathrm{MeV}$. Although the broken spectra would be little debated due to system errors from multiple spacecraft, it is hard to obtain a completely spectra for a large energy span from an individual spacecraft. So we hopefully do a simulation to obtain an entire spectra, which can cover the energy range from thermal energy below $\sim 0.1 \mathrm{MeV}$ to high energy beyond $\sim 10 \mathrm{MeV}$. Fig. 1 shows a schematic diagram of the converged shocks model. The left reflective wall represents a CME, which produces a shock by a bulk flow speed of $u 0_{2}$. The right reflective wall represents the Earth, which informs a bow shock by an opposite bulk flow speed of $u 0_{1}$. Their relative speed between two bulk flows is equal to $u=\left|u 0_{1}\right|+\left|u 0_{2}\right|$, which can equivalently be taken as the relative movement between two reflective walls with opposite velocities $u 0_{1}, u 0_{2}$ in the laboratory reference frame. Similarly, we can take both downstream bulk flow speeds with the same velocity zero in the laboratory reference frame. This model describes the double shocks interaction occurred on the 13 December 2006 nearby Earth. According to the Wind magnetic cloud list, the cloud axis direction is $\theta=27^{\circ}, \phi=85^{\circ}$ in GSE coordinates [17]. In this model, we define the bulk flow direction to the interplanetary magnetic field (IMF) direction with an oblique factor of $\cos (\theta)$. So we take the relative speed between two bulk flows as value for $\sim 1600 \mathrm{~km}^{-1}$ aligned to the IMF. Both two shocks are produced by the same bulk flow speed value for $\left|u 0_{1}\right|=\left|u 0_{2}\right|=\sim 800 \mathrm{~km}^{-1}$, but with opposite direction in the laboratory frame. Also both two reflective walls produce shocks propagating with opposite velocities of $v_{s h 1}$ and $v_{s h 2}$, respectively.

In this MC method, we apply an initial number density of particles $n_{0}$ obeying a Maxwellian distribution with a random thermal velocity $v_{0}$ in the unshocked upstream region. Initial particles with their upstream bulk flow speeds move to their corresponding reflective walls in the both sides of the simulation box. Each bulk flow is reflected and forms the higher shocked densities as downstream region in each side of the simulation box. With the downstream density achieving to a saturation, the shock fronts smoothly evolute forward to the middle of the box with shock velocity $V_{s h 1}$ and $V_{s h 2}$, respectively. When the two shock fronts propagate more and more close, then the two precursors have an interaction with the time at the middle of the simulation box. It is this processes the particles are able to gain their additional energies by crossing two shock fronts.

In this simulation box, the continuous bulk flow from the middle of the box with opposite directions enter into the box along to the two boundaries of the simulation box. One bulk flow forms a CME shock in the left boundary, another bulk flow forms a bow shock on the right boundary. 


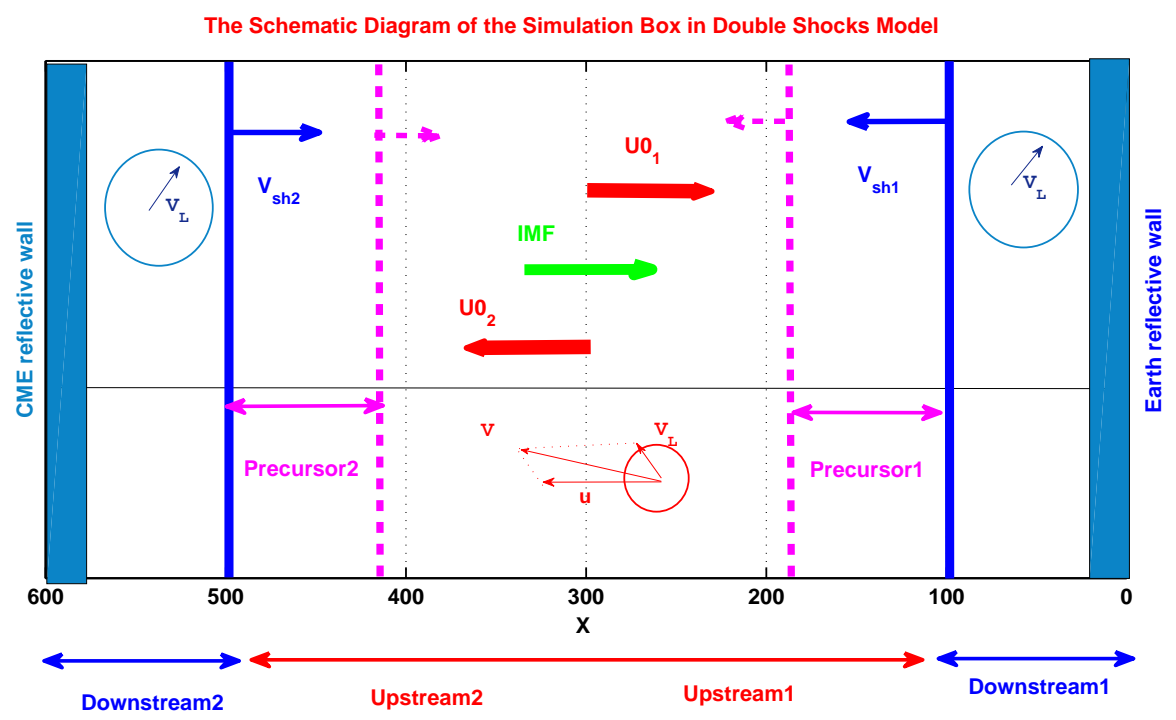

Figure 1: A schematic diagram of the converging shock model.

With the shock formations, the particles are accelerated from each shock front. With the shock propagations forwarding to the middle of the box, the precursor regions are mixed. This interaction of the precursor regions ahead of two shocks make the energetic particles either re-accelerate or de-accelerate at the end of the simulation. It is this double effect of the energetic particles is responsible to the formation of the energy spectral "break" at the range of $1 \mathrm{MeV}-10 \mathrm{MeV}$.

\section{Model 2}

This model describes the "twin-shock" interactions on 2012 May 17 GLE event at the interplanetary space less than $5 R_{\odot}$ (here, $R_{\odot}$ is the radii of the Sun). Since the active region (AR) is located at W88 from the Earth, the event was a backside event for STEREO-B. The propagation directions of the two CMEs are also shown in coronagraph observations made by SOHO/LASCO. While the two CMEs were clearly seen from STEREO-B and SOHO, they overlapped and appeared to be propagating together and were hardly distinguishable from the STEREO-A observations. Shen et al. [6] use the Graduated Cylindrical Shell (GCS) model to give the height $h$ of the two CMEs at different times and the parameters of the longitude $\phi$, the latitude $\theta$, and others. The differences of $\delta \theta$ and $\delta \phi$ between the two CMEs are $11^{\circ}$ and $31^{\circ}$. This means that there are two distinct CMEs in this event and they have different but close propagation directions. According to the calculated list of the hight of the two-CMEs at different times, they gave the hight $h_{1}=4.5 R_{\odot}$ for CME1 and the hight $h_{2}=4.82 R_{\odot}$ for CME2 at the time of 02:00UT. The difference of the hights of the two CMEs is $0.32 R_{\odot}$. They also used the relationship between the hight of CMEs and the propagation time [7] to obtain the speeds of the two CMEs, which are $1258 \pm 352 \mathrm{~km} / \mathrm{s}$ and $1539 \pm 352 \mathrm{~km} / \mathrm{s}$ for CME1 and CME2, respectively. 


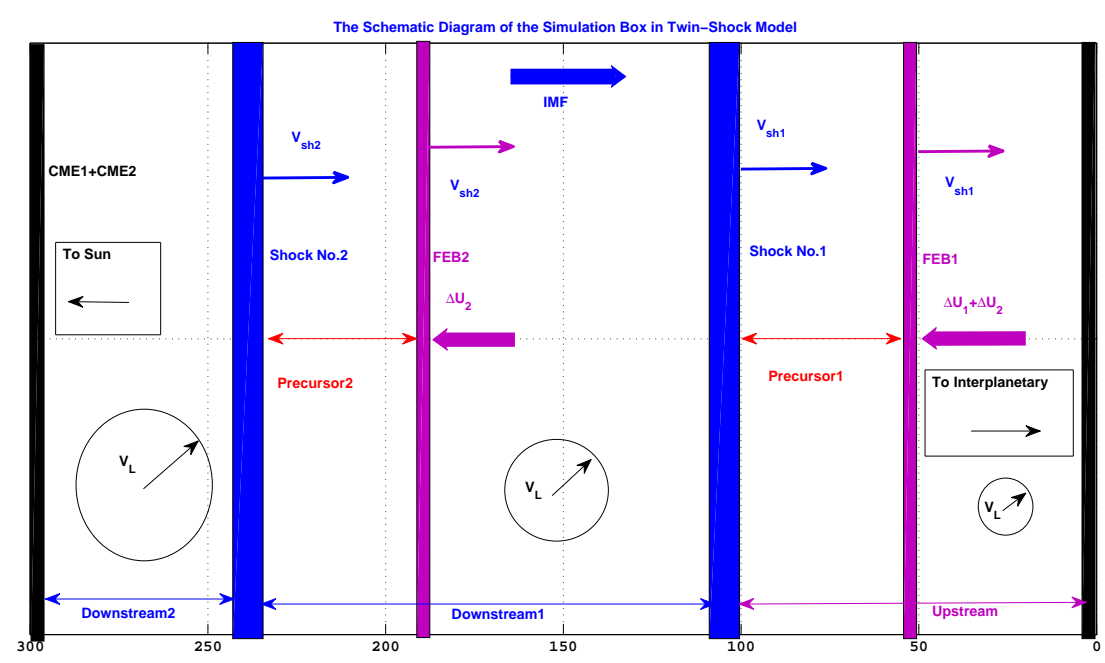

Figure 2: A schematic diagram of the pileup shock model.

Fig. 2 shows a schematic diagram of the pileup shock model. The left reflective wall represents CMEs and produce the shock No.1 and No.2 propagating from the left of the simulation box to the right. After a duration, the shock No.1 propagating into the center of the simulation box, the second CME appears at the right reflective wall and produces the shock No.2. The shock No.2 also propagates into the simulation box with a relative bulk speed $\Delta U_{2}$ to the shock No.1, which indicate by the two blue vertical bars at the simulation box in Fig.2. Precedently, the upstream bulk flow speed of the shock No.1 is $\Delta U_{1}$ and the downstream bulk flow speed is zero at the rest reference frame of the shock No.1. When the shock No.2 appears at the left boundary, then there is an interaction with the shock No.1 with a relative bulk flow speed $\Delta U_{2}$. So at the rest reference frame of the shock No.2, both of the upstream and downstream bulk speeds of the shock No.1 add an increment of $\Delta U_{2}$ become to be the $\Delta U_{1}+\Delta U_{2}$ and $\Delta U_{2}$, respectively. Correspondingly, the upstream and downstream bulk speeds of the shock No.2 become the $\Delta U_{2}$ and zero. In the twin-shock scenarios, since there is a relative bulk speed $\Delta U_{2}$ between the two shocks, the downstream bulk flow of the shock No.1 is compressed by the shock No. 2 while the two CMEs commencing a collision. So the heating and accelerated ions at the downstream of the shock No.1 can be re-acceleration by Fermi acceleration processes at the shock No.2. We perform a Mote-carlo code to simulate the twin-shock scenario in detail for investigating the alteration of the energy spectrum undergoing the "twin-CMEs" collisions.

Initially, the upstream bulk flow of the shock No.1 remains the bulk speed with $\Delta U_{1}$ until there is a collision between the CME1 and the CME2 at the left boundary of the simulation box. Here, we set the total simulation time is $t_{\max } / 2$. At the half of the total simulation time, we set the two CMEs begin a collision at the left reflective wall of the simulation box. Then, during the next half of the total simulation time $t_{\max }$, the particles will undergo re-acceleration processes on the collided region between the twin-shock. We suggest the CME1 would produce the shock No.1 forming a normal power-law energy spectrum, but the CME2 would produce the shock No.2 enhancing the existing normal power-law energy spectrum. In this "twin-shock" scenarios, the twin-CME shocks 
have a collision with the same direction from the source to the collided location. Therefore the shock No.2 produce a positive effect on the shock No.1 resulting an enhancement for the existing energy spectrum. We predict there would appear a "concave" energy spectrum at a certain energy range, which would be identified by performing the "twin-shock" simulation on the 2012 May 17 SEP event.

\section{Comparison of the energy spectra}
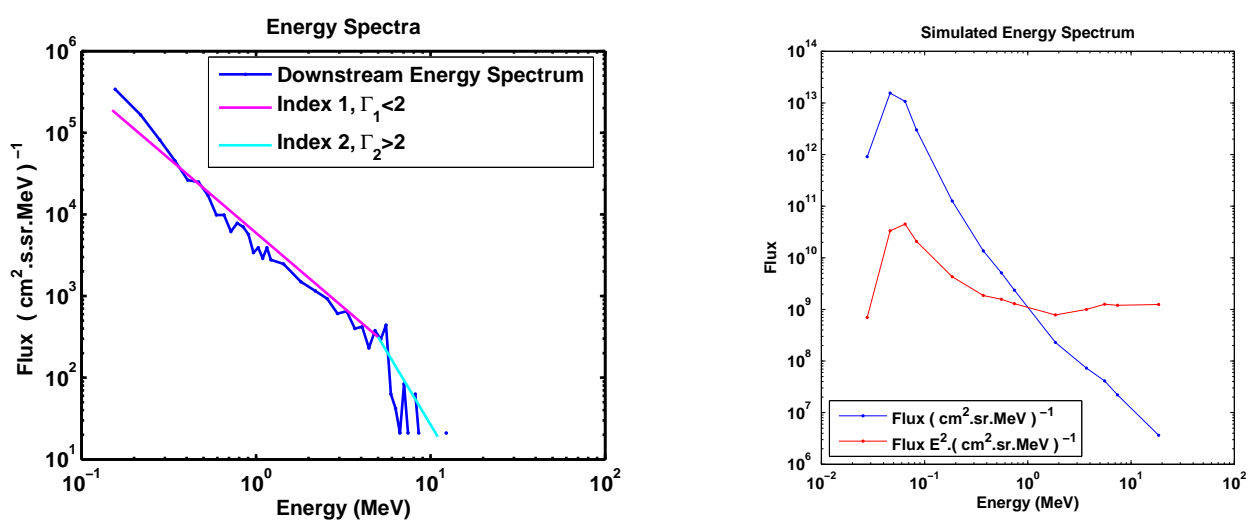

Figure 3: The left plot is the simulated energy spectrum of the converging shock model. The pink line and cyan line show an energy spectral "break" at $\sim 5 \mathrm{MeV}$. The right plot shows the spectrum of the pileup shock model.

Fig. 3 shows a comparison of the simulated energy spectra between the converging shock model and pileup shock model. The left plot represents the simulated energy spectra calculated from the simulation box at the end of the simulation. The solid curve shows an energy spectral "break" at $\sim 5 \mathrm{MeV}$. The pink line represents the hard energy spectrum at low energy range with an index less than 2, and the cyan line represents the soft energy spectrum at high energy range with an index more than 2. The "break" indicates that there exists a double power-law spectrum. The right plot shows the simulated energy spectrum of the pileup shock model with two different presentations for particle fluxes. The blue curve represents the particle fluxes with the normal representation, the red curve represents the special energy spectrum by using a factor of the square of the energy times the flux. From the right plot, the energy spectrum covers the total energy range from $30 \mathrm{keV}$ to $20 \mathrm{MeV}$. There is a maxwellian thermal peak at about $\sim 40 \mathrm{keV}$. Then the suprethermal particles "tail" is shown at the energy range from the $\sim 70 \mathrm{keV}$ to $400 \mathrm{keV}$. At the energy range from the $400 \mathrm{keV}$ to $\sim 2 \mathrm{MeV}$, the energy spectrum shows a softer power-law property with an index more than 2 . Comparably at the energy range from the $\sim 2 \mathrm{MeV}$ to $\sim 20 \mathrm{MeV}$, the energy spectrum shows a harder power-law property with an index less than 2 . The red curve can clearly show that "ankle-like" property in the total energy spectrum. According to our calculation from the red curve, we suggest that there is the "ankle" point at $\sim 2 \mathrm{MeV}$. We predict there exists a "ankle" point at $\sim 2 \mathrm{MeV}$ due to the twin-shock interaction take a positive effect on the pre-acceleration particles resulting a hard energy spectral shape. 


\section{Summaries and Conclusions}

On the one hand, we simulate the converged two shocks for obtaining the proton spectrum directly. Comparing with the observed energy spectrum, the simulated energy spectrum exhibits the consistent energy spectral "break" at $\sim 5 \mathrm{MeV}$. Applying the double shocks model, we obtain the extended energy spectrum with the highest energy "tail" up to $\sim 10 \mathrm{MeV}$, and also we identify that the energy spectrum is indeed broken at $\sim 5 \mathrm{MeV}$.

On the other hand, we simulate the pileup shock model for predicting the proton spectral property. We obtain the total energy spectrum covering the energy range from $30 \mathrm{keV}$ to $20 \mathrm{MeV}$. We actually find the simulated energy spectrum exhibits an "ankle" structure at the energy point of $\sim 2 \mathrm{MeV}$.

With the comparison of the previous converging double-shock model, in which we have investigated an energy spectrum "break" at $\sim 5.5 \mathrm{MeV}$, why do the pileup shock model would produce an "ankle" energy spectrum? There would be some reasons: (i) According to the diffusive shock acceleration theory, the acceleration efficient is determined by the diffusive coefficient. The attainable highest energy particle is depended on the diffusive length of particles scaled by the size of the precursor region. At the converging shocks, the precursor region size will be shorten and fewer and fewer high energy particles gain energies resulting a softer energy at the high energy "tail". (ii) But in the pileup shock model, the twin-shock interaction can extend the precursor region size and enhance the existing accelerated particle distribution. The pre-accelerated particles due to the first shock, penetrate into the second shock for re-accelerating and modifying the existing power-law energy spectrum to a harder energy spectrum. These processes can lead to an "ankle" shape on the energy spectrum at a certain energy range. (iii) Furthermore, whatever in the converging shock model or the pileup shock model, both the diffusive particles can extend the energy spectrum up to a few decades of $\mathrm{MeV}$ in their high energy "tails". The specific energy spectral shape would be desponded on their collision forms, in which the important parameter of the precursor size will be decided.

Therefore, we present some possibilities for forming an extended energy spectrum and producing a varied slope in different double- shock models. First of all, the converging shock interaction would provide more kinetic energy injecting into the particles acceleration. The high efficient injection rate excited by amplified magnetic turbulence from the converged region make the extended energy spectrum be possible. Secondly, the pileup shock model provide the opportunities of the re-accelerated processes for the existing energy spectrum occur on the first shock. With the expended precursor region, more and more population of the particles can participate into the twinshock system, the "ankle" slope of the energy spectrum would be produced due to the enhancement magnetic turbulence between the twin shocks. We have proved that the converging shock model taken a negative effect on the accelerated particles and produce an energy spectral "break". We also investigate that a positive effect on particle acceleration in pileup shock model can produce an energy spectral "ankle" propertiy. 


\section{6. acknowledgements}

This work is supported by the Xinjiang Natural Science Foundation No. 2019D01A100, and Xinjiang Heaven Lake Hundred-Talent Program. The present work is also supported by State Key Laboratory of Space Weather, Chinese Academy of Sciences, CAS Key Laboratory of Solar Activity, National Astronomical Observatories, the Key Laboratory of Modern Astronomy and Astrophysics (Nanjing University), Ministry of Education, China Scholarship Council (CSC), and NSFC (No.11673056). Simultaneously, authors thank the support from the Supercomputer Center of University of Arizona. In addition, the authors appreciate Profs. J. R. Jokipii, Joe Giacalone and his group in the University of Arizona, Profs. Hongbo Hu at IHEP, Prof Chuan Li at NJU, Prof Jun Lin at YNAO and Profs Yuming Wang, Chenlong Shen and Quanming Lu at USTC in Chinese Academy of Sciences, Profs. Zhengming Sheng, Min Chen and Suming Wen at Shanghai Jiao Tong University for their many helpful discussions.

\section{References}

[1] Chen, C., Wang, Y., Shen, C. \& et al. 2011, J. Geophys. Res., 116, A12108

[2] Ding, L. G., Jiang, Y., Zhao, L. \& Li, G. 2013, ApJ, 763, 30

[3] Ding, J. Y., Hu, Y. Q. \& Wang, J. X. 2006, SoPh, 235, 223

[4] Li, G., Moore, R., Mewaldt, R. A., Zhao, L., \& Labrador, A. W. 2012, SSRv, 171, 141

[5] Liu, C., Lee, J., Karlicky, M., \& et al. 2009, ApJ, 703, 757

[6] Shen, C., Li, G., Kong, X., Hu, J., Sun, X. D., et al. 2013, ApJ, 763,114

[7] Thernisien, A. F. R., Vourlidas, A., \& Howard, R. A. 2009, SoPh, 256, 111-130

[8] Wang, X. et al., 2013, ApJS, 209,18

[9] Wang, X. et al., 2011, Astron. Astrophys., 530, A92

[10] Wang, X. et al., 2012, Res. Astron. Astrophys., 12, 1535-1548

[11] Wang, X. et al., 2016, Res. Astron. Astrophys., 16, 32

[12] Wang, X. et al., 2017, ApJ, 842, 74

[13] Wang, X. et al., 2019, ApJ, 885, 66

[14] Zhukov, A. N., \& Veselovsky, I. S. 2007, ApJL, 664, 131

[15] Ellison, D. C., Möbius, E. \& Paschmann, G., 1990, ApJ,352,376-394

[16] Knerr, J. M., Jokipii, J. R. \& Ellison, D. C., 1996, ApJ, 458,641-652

[17] Liu, Y., Luhmann, J. G., Müller-Mellin, R., et al., 2008, ApJ, 689, 563.

[18] Mewaldt, R. A., et al. ,2008, ,Proceedings of the 30th ICRC(Mexico), 1, 107-110. 